\title{
High-Index Dielectric Metasurfaces Performing Mathematical Operations
}

\author{
Andrea Cordaro, ${ }^{\dagger, \ddagger \odot}$ Hoyeong Kwon, ${ }^{\S}$ Dimitrios Sounas, ${ }^{\S, \|}$ A. Femius Koenderink, ${ }^{\ddagger}$ Andrea Alù, ${ }^{\S, \perp_{\odot}}$ \\ and Albert Polman* $*$;० \\ ${ }^{\dagger}$ Van der Waals-Zeeman Institute, Institute of Physics, University of Amsterdam Science Park 904, 1098 XH Amsterdam, The \\ Netherlands \\ ${ }^{\ddagger}$ Center for Nanophotonics, AMOLF, Science Park 104, 1098 XG Amsterdam, The Netherlands \\ ${ }^{\S}$ Department of Electrical and Computer Engineering, The University of Texas at Austin, Austin, Texas 78712, United States \\ "Department of Electrical and Computer Engineering, Wayne State University, Detroit, Michigan 48202, United States \\ ${ }^{\perp}$ Photonics Initiative, Advanced Science Research Center, City University of New York, New York, New York 10031, United States
}

Supporting Information

ABSTRACT: Image processing and edge detection are at the core of several newly emerging technologies, such as augmented reality, autonomous driving, and more generally object recognition. Image processing is typically performed digitally using integrated electronic circuits and algorithms, implying fundamental size and speed limitations, as well as significant power needs. On the other hand, it can also be performed in a low-power analog fashion using Fourier optics, requiring, however, bulky optical components. Here, we introduce dielectric metasurfaces that perform optical image edge detection in the analog domain using a subwavelength geometry that can be readily integrated with detectors. The metasurface is composed of a suitably engineered array of nanobeams designed to perform either first- or second-order spatial differentiation. We experimentally demonstrate the second-derivative operation on an input image, showing the potential of all-optical edge detection using a silicon metasurface geometry working at a numerical aperture as large as 0.35 .

KEYWORDS: Analog computing, metasurface, Fano resonance, spatial differentiation, image processing, edge detection
$\mathrm{T}$ he amount of data that is being globally created, processed, and stored is increasing at a remarkable pace. Furthermore, the advent of new technologies, such as augmented reality, autonomous driving, and many other emerging techniques, requires on-the-fly processing of large data files, such as images, at an increasing rate. Image processing is usually performed digitally, but the speed and power consumption limits of standard microelectronic components have become a true bottleneck. Analog optical processing provides a promising route that may overcome these limitations.

The idea of computing optically can be traced back to the early 1960s prompted by pioneering work that exploited concepts of Fourier optics. ${ }^{1-3}$ These elegant all-optical solutions, however, require bulky optical components that are not integrable into a larger nanophotonic system and have remained unpractical. The unprecedented control of light propagation over a subwavelength thickness that has been recently enabled by optical metasurfaces opens entirely new opportunities for analog optical computing. ${ }^{4-9}$ In fact, "computing metasurfaces" may benefit from the speed and low power consumption of optics while being amenable to on- chip integration, thus enabling hybrid optical and electronic data processing on a single chip.

In this work, we design and realize optical metasurfaces composed of dielectric nanobeams that are illuminated by light polarized along the beams' direction. We tailor the spatial dispersion of the metasurfaces by controlling the leaky modes guided along the surface. ${ }^{10-12}$ When the frequency and inplane wave vector of incident light match one of these quasiguided modes, an asymmetric Fano line shape appears in the transmission spectrum ${ }^{13-15}$ (see Figure 1), due to interference with the broad Fabry-Pérot resonance determined by the thickness and fill fraction of the structure. Figure 1 shows the simulated transmission spectra of an array of dielectric nanobeams (width $w=182 \mathrm{~nm}$, height $h=123 \mathrm{~nm}$, pitch $p$ $=250 \mathrm{~nm}$, and refractive index $n=4$ typical for $\mathrm{Si}$ ) for incident angles ranging from 0 to 0.3 radians $\left(17^{\circ}\right)$. Due to the Fano interference, the transmission swings from 0 to unity within a narrow bandwidth. The sharp response in frequency

Received: June 18, 2019

Revised: October 9, 2019

Published: November 1, 2019 


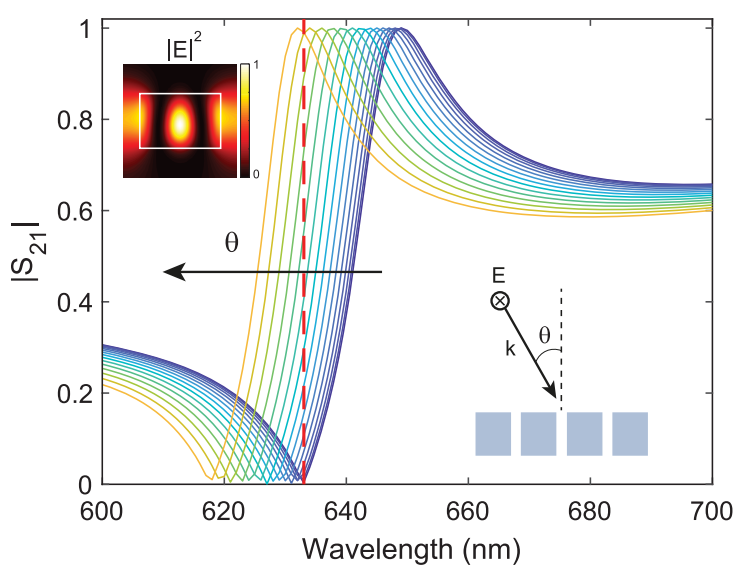

Figure 1. Fano-resonant metasurface transmission spectra. Simulated transmission spectra of a metasurface consisting of an array of dielectric nanobeams (width $w=182 \mathrm{~nm}$, height $h=123 \mathrm{~nm}$, pitch $p$ $=250 \mathrm{~nm}$, and refractive index $n=4)$ as the incident angle is changed from 0 (blue line) to $0.3 \mathrm{rad}$ (yellow line) in 15 steps. The red dashed line indicates the wavelength of operation $(\lambda=633 \mathrm{~nm})$. Insets: (bottom right) schematic of the proposed structure showing incoming light polarization; (top left) electric field amplitude profile within a unit cell at the resonant wavelength.

corresponds to strong nonlocality: the spectrum is largely dependent on the incident angle, and the transmission minimum shifts from $\lambda=633 \mathrm{~nm}$ to $\lambda=618 \mathrm{~nm}$ over the simulated angular range. The strong amplitude variation in transmission and the sensitivity to the incoming $k$-vector are often undesirable features of resonant metasurfaces, yet here these features enable the use of the metasurface as a Fourier spatial filter, and tailor with large flexibility its angular transmission response, or transfer function. In fact, by tuning the dispersion of the quasi-guided mode resonance, as well as the Fano line-shape asymmetry and line width, it is possible to design an optimized transfer function for a specific excitation wavelength (see the Supporting Information). ${ }^{15-21}$

Starting with the case of second-order spatial differentiation, if $f(x)$ is an arbitrary wave input signal, then its second derivative $\frac{\mathrm{d}^{2} f(x)}{\mathrm{d} x^{2}}$ equals $-k_{x}^{2} f\left(k_{x}\right)$ in the spatial domain, where $\tilde{f}\left(k_{x}\right)$ is the Fourier transform of $f(x)$. Therefore, second-order differentiation can be achieved by a metasurface that imparts a parabolic transfer function and a slowly varying phase to the spatial frequencies composing the input signal. ${ }^{22,23}$

Figure $2 \mathrm{a}$ shows the simulated transmission amplitude $\left|S_{21}\right|$ and phase $\arg \left(S_{21}\right)$ as a function of the in-plane wave vector $k_{x}$, normalized by the free space wave vector $k_{0}$ at the design wavelength $\lambda=633 \mathrm{~nm}$ for the metasurface presented in Figure 1. In this design, optimized to perform second-order differentiation, the angular response is close in amplitude to the ideal parabolic shape. The phase response shows a variation of approximately $0.9 \pi$, deviating at high angles from the ideal constant phase response but still providing a close-toideal second-derivative response.

Our metasurface design has two key features that distinguish it from earlier designs. First of all, the metasurface operational numerical aperture is large $(\mathrm{NA} \approx 0.35)$. This feature enables processing images with high spatial content and hence a resolution close to the diffraction limit. Moreover, it allows for direct implementation into standard imaging systems with similar NA, for instance, by placing the metasurface right in front of a charge-coupled device (CCD) detector array, without needs for additional imaging lenses (see the Supporting Information). This is a major advance over previously explored spatial differentiation schemes ${ }^{6-8}$ that operate at an NA that is $\sim 25$ times smaller than what we demonstrate here. Second, the transmission in our design reaches unity at large wave vectors, enabling close-to-ideal image transformation efficiency, significantly larger than earlier attempts at realizing image processing metasurfaces.

Next, we use the optimized transfer function to numerically test how well the ideal second-order differentiation is approximated by our realistic metasurface design. Figure 2c shows the calculated response for rectangular and sinusoidal input functions shown in Figure $2 b$. The metasurface output clearly shows the edges of the rectangular input profile and flips the sinusoidal input function as expected (see the Supporting Information for the real and imaginary part of the metasurface output and the comparison with its ideal counterpart). It is also possible to process arbitrary $2 \mathrm{D}$ images by performing the second derivative line by line. The edges of one of our institutions' logos are clearly visible in Figure 2c. Notice that differentiation is performed only along the $x$-axis for this 1D geometry; hence, the edges along the same direction are not detected.

To illustrate the flexibility of the metasurface image processing concept, Figure $2 \mathrm{~d}$ shows the optimized transmission for a metasurface performing first-order differentiation, which corresponds to the transfer function $S_{21}\left(k_{x}\right)=\mathrm{i} k_{x}$ in the Fourier domain. In order to achieve such an operation with odd symmetry in space, we designed an asymmetric metasurface composed of an array of Si nanobeams with a unit cell ( $p$ $=300 \mathrm{~nm})$ consisting of nanobeams with two different widths $\left(w_{1}=48 \mathrm{~nm}, w_{2}=96 \mathrm{~nm}, h=165 \mathrm{~nm}\right.$, and gap between the nanobeams of $53 \mathrm{~nm}$ ), placed on a thin silicon layer (thickness $t=35 \mathrm{~nm}$ ) on a semi-infinite $\mathrm{Al}_{2} \mathrm{O}_{3}$ substrate. Indeed, by Lorentz reciprocity, it is easy to prove that the unit cell has to be asymmetric both along the direction of propagation and transversally. Furthermore, the asymmetry in the phase response of the transfer function can be tuned with $t$, enabling the required $\pi$ phase jump at $k_{x}=0$ (see the Supporting Information). ${ }^{5}$ In this case, the experimental optical constants (including losses) for the two materials have been used in the simulations. ${ }^{24,25}$ The simulated transfer function amplitude shows a linear behavior over a wave vector range up to $k_{x} / k_{0}=$ $0.1\left(6^{\circ}\right)$, above which it gradually bends away from the ideal case. For large angles, the transmission saturates below unity due to intrinsic absorption in Si. Figure 2e,f shows the calculated metasurface output for rectangular, parabolic, and triangular input functions. The input slope changes, and a nearly linear derivative for the parabola is clearly resolved. Furthermore, processing the logo results in clear detection of the edges in the $x$-direction consistently with first-derivative operation.

In order to demonstrate second-order differentiation experimentally, we fabricated a $1 \mathrm{D}$ array of $\mathrm{Si}$ nanobeams on a $0.46 \mathrm{~mm}$ thick sapphire $\left(\mathrm{Al}_{2} \mathrm{O}_{3}\right)$ substrate, using a combination of electron beam lithography and reactive ion etching (see the Methods). Figure 3a depicts a scanning electron microscopy (SEM) image of the fabricated structure showing high uniformity over a large area. The optimized dimensions for this design are $w=206 \mathrm{~nm}, h=142 \mathrm{~nm}$, and $p$ $=300 \mathrm{~nm}$. Figure $3 \mathrm{~b}$ shows a cross section of the nanobeams. The etched Si side walls are straight to within $\sim 20 \mathrm{~nm}$, and a thin residual Si layer (thickness $t \approx 22 \mathrm{~nm}$ ) is intentionally left 


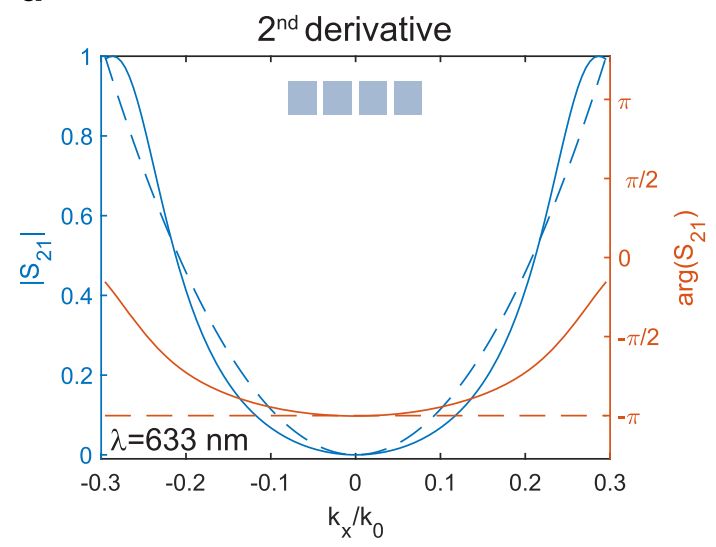

d

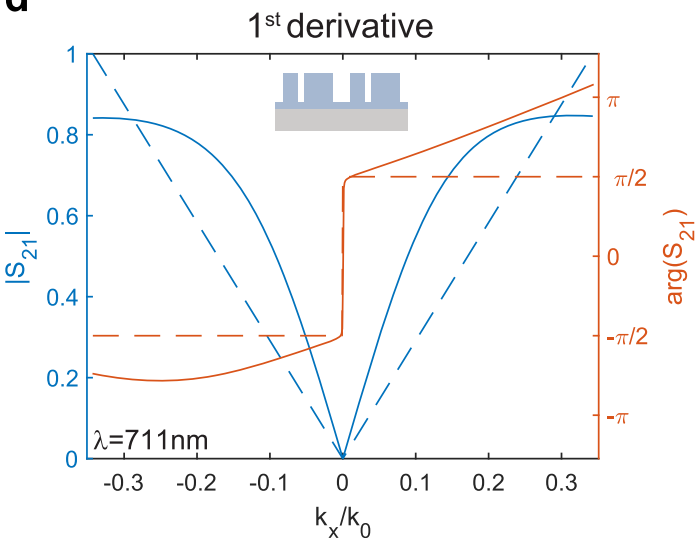

b

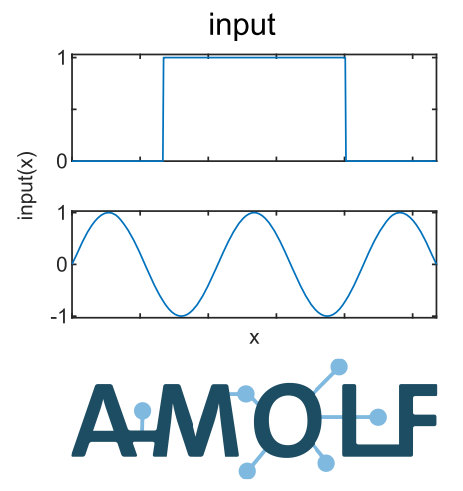

e

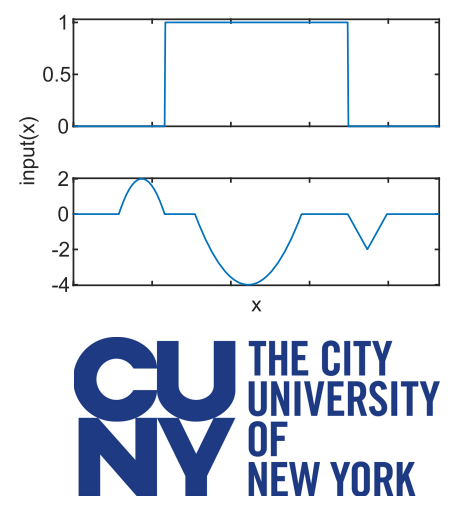

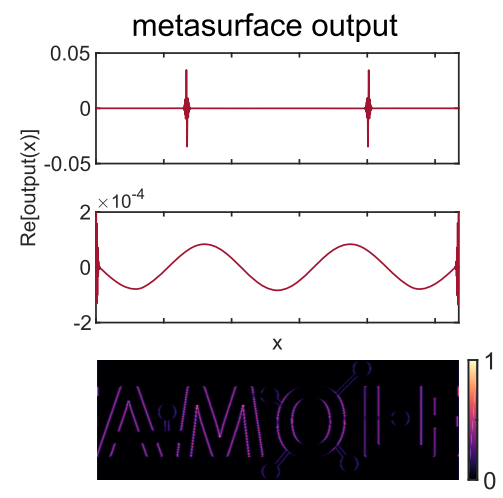

$\mathbf{f}$

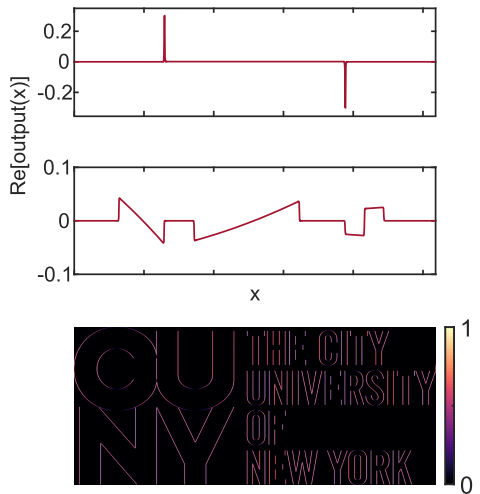

Figure 2. Simulated transfer functions of dielectric metasurfaces performing first- and second-order spatial differentiation. (a) Transmission amplitude $\left|S_{21}\right|$ (solid blue line) and phase $\arg \left(S_{21}\right)$ (solid orange line) for the metasurface optimized for second-derivative operation (sketched in the inset) at $\lambda=633 \mathrm{~nm}$. The simulated transfer function is compared to the ideal case (dashed lines). The transmission reference plane is set such that the transmission phase at normal incidence equals $-\pi$. (b) Rectangular and sinusoidal input functions and 2D image that are used to numerically test the metasurface operation. The signal is discretized into 1000 pixels with individual pixel size set such that the Nyquist range matches the operational range in $k$-space of the metasurface. (c) Metasurface output for the input in part b. For the 2D image, differentiation is performed line by line along the $x$-axis. $(\mathrm{d}-\mathrm{f})$ Same as parts a $-\mathrm{c}$ but for first-derivative operation (metasurface geometry sketched in the inset) compared to the ideal case (dashed lines) at $\lambda=711 \mathrm{~nm}$. The transmission reference plane is set such that the transmission phase at normal incidence is 0 . The AMOLF and CUNY logos are reproduced with permission.

between the pillars. This layer is essential to achieve optimum transmission for large wave vectors.

Figure $4 \mathrm{a}$ shows the measured transmittance $\left(T=\left|S_{21}\right|^{2}\right)$ spectra as the incident angle is changed from 0 to $25^{\circ}$. In agreement with the simulated data in Figure 1, the Fano resonance shifts to shorter wavelengths as the angle is increased. The transmittance minimum is observed at $\lambda=$ $726 \mathrm{~nm}$ for normal incidence and amounts to $2.2 \%$, the residue attributed to minor fabrication imperfections. Figure $4 \mathrm{~b}$ shows the transmittance as a function of the in-plane wave vector at $\lambda$ $=726 \mathrm{~nm}$, derived from the data in Figure 4a. The corresponding transmission amplitude $\left(\left|S_{21}\right|\right)$ derived from the data is also plotted, along with the ideal parabolic amplitude response. The overall trend with increasing transmittance as a function of angle is well reproduced experimentally, with a significant residual transmittance at normal incidence and a maximum amplitude at the largest angle of 0.84 , which is mostly determined by the absorption in Si. Employing alternative high-index materials could further enhance the transmission for large angles.

The inset in Figure $4 \mathrm{~b}$ shows the metasurface transfer function amplitude in wavenumber space taken with a Fourier microscope (see the Supporting Information). These measure- ments clearly show the $1 \mathrm{D}$ nature of the metasurface operation. In fact, low $k_{x}$ spatial components are suppressed also for a wide range of $k_{y}$. Hence, the second-order differentiation is experimentally performed in a line-by-line fashion, in agreement with the numerical calculation shown in Figure 2.

Finally, we experimentally investigate the second-derivative operation of the Si metasurface when an image is projected onto the sample. To this end, we fabricated one of our institutions' logos using a Cr pattern on glass (Figure 5a). We first project the image onto the metasurface using off-resonant illumination $(\lambda=750 \mathrm{~nm})$ and then image the metasurface output onto a CCD imaging camera (Figure $5 b$ ) (see the Supporting Information); the contrast of the input object is clearly maintained. On the other hand, for resonant illumination at $\lambda=726 \mathrm{~nm}$ (Figure 5c), the edges are clearly resolved in the transformed image. As expected, no edge contrast is observed for features along the $x$-direction.

To study the edge profile in a quantitative manner, Figure $5 \mathrm{~d}$ (red curve) shows a line profile taken along the horizontal direction in the processed image (red dashed line in Figure 5c). These data are compared to the calculated output profile assuming an ideal parabolic transfer function (blue curve in 


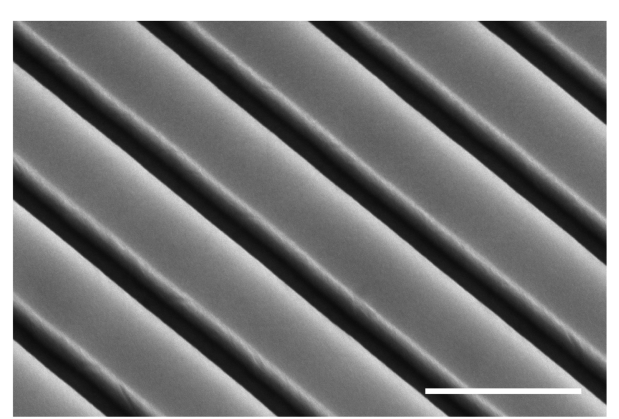

b

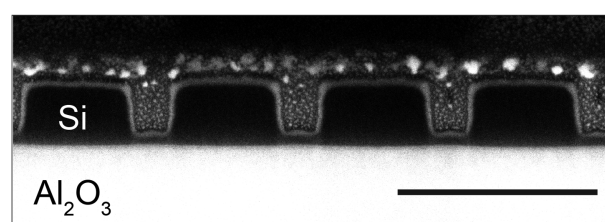

a

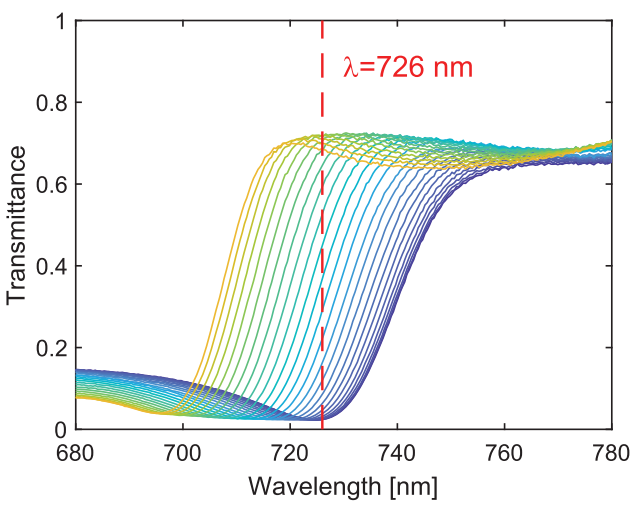

b

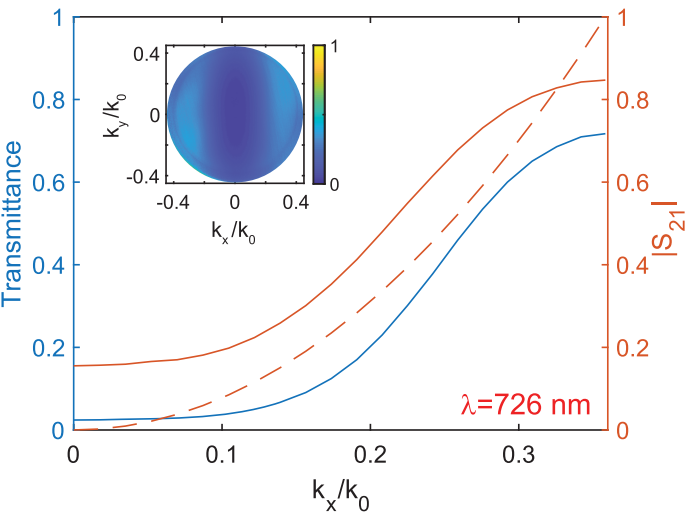

Figure 4. Experimental metasurface transmission. (a) Measured transmission spectra of the metasurface in Figure 3 as the angle of incidence is increased from 0 (blue line) to $25^{\circ}$ (yellow line) in 25 steps. (b) Measured transmittance (blue line) and corresponding calculated (transmittance $=\left|S_{21}\right|^{2}$ ) transmission amplitude $\left|S_{21}\right|$ (orange solid line) as a function of incident in-plane wave vector $k_{x} / k_{0}$ at $\lambda=726 \mathrm{~nm}$. The dashed orange line shows the ideal parabolic transfer function for $\left|S_{21}\right|$. Inset: measured metasurface transfer function amplitude at $\lambda=726 \mathrm{~nm}$ (see the Supporting Information for the description of the setup used).

optical and electronic computing that operates at low cost, low power, and small dimensions.

Methods. Fabrication. Metasurfaces. The structures were fabricated by electron beam lithography (EBL) as follows:

(1) c-Si on $\mathrm{Al}_{2} \mathrm{O}_{3}$ substrates were acquired from MTI Corp.

(2) The substrate was cleaned in base piranha, and the c-Si was etched to the final metasurface thickness via reactive ion etching (RIE) using a two-step process employing $\mathrm{Cl}_{2}, \mathrm{HBr}$, and $\mathrm{O}_{2}$.

(3) The substrate was cleaned again in base piranha, and a $200 \mathrm{~nm}$ thick layer of CSAR 62 (AR-P 6200, 9\% in anisole) positive-tone resist was spin-coated and baked for $2 \mathrm{~min}$ at $150{ }^{\circ} \mathrm{C}$.

(4) Lines were fabricated in the CSAR layer by exposure using a Raith Voyager lithography system $(50 \mathrm{kV}$, dose $145-150 \mu \mathrm{C} / \mathrm{cm}^{2}$ ) and development in pentyl-acetate $(60 \mathrm{~s})$ and $o$-xylene $(10 \mathrm{~s})$.

(5) The pattern was then transferred into the c-Si by a twostep $\mathrm{RIE}$ process employing $\mathrm{Cl}_{2}, \mathrm{HBr}$, and $\mathrm{O}_{2}$.

(6) The sample was finally cleaned in anisole at $65{ }^{\circ} \mathrm{C}$ followed by an acid piranha cleaning.

Images. 


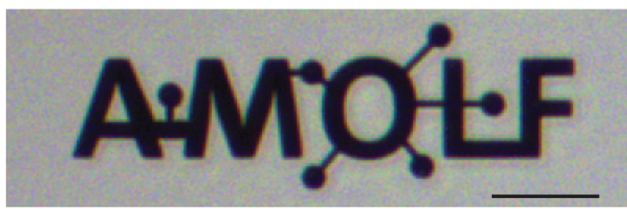

b

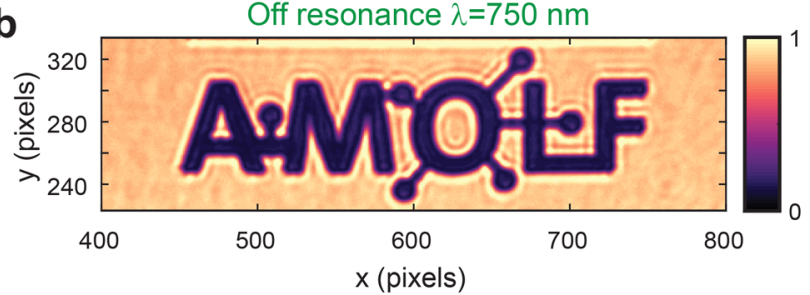

C

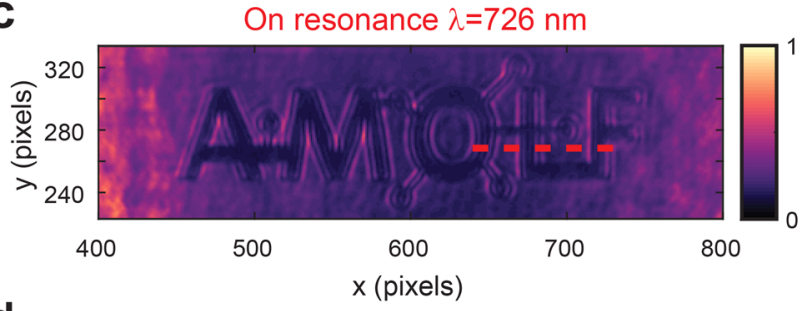

d

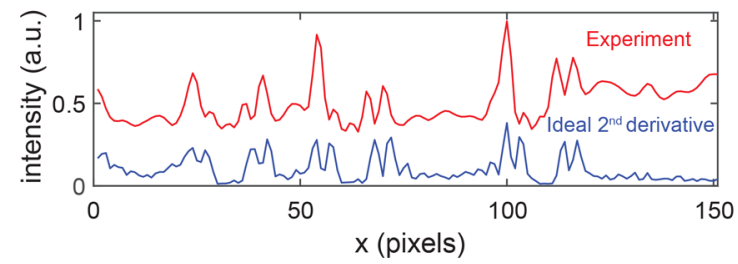

e

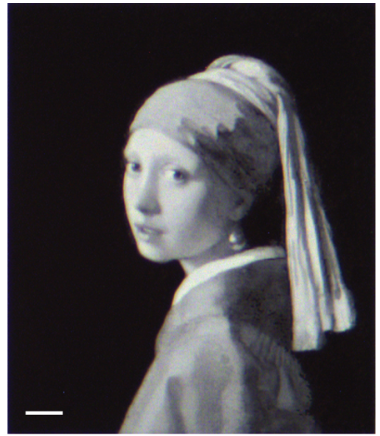

f

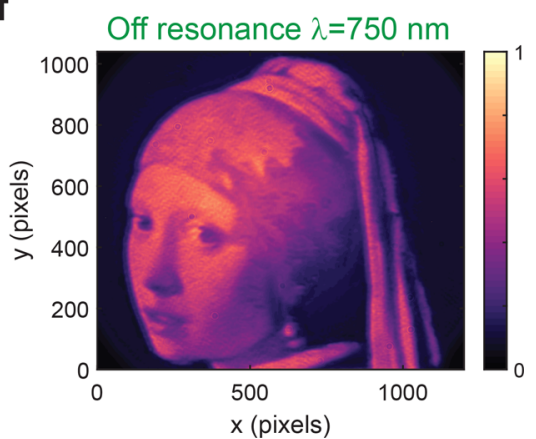

g

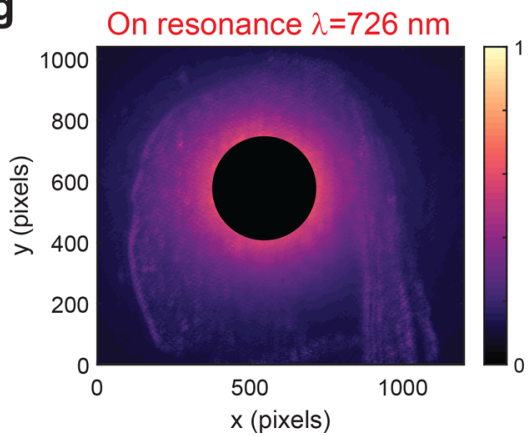

Figure 5. Experimental second-order image differentiation. (a) Optical microscopy image of the input object; the scale bar is $20 \mu \mathrm{m}$. (b, c) Optical microscopy image of the metasurface output for resonant $(\lambda=726 \mathrm{~nm})$ and off-resonant $(\lambda=750 \mathrm{~nm})$ illumination. (d) Cross-cut through part $b$ (red line) averaged over 8 pixels along $y$, compared to ideal differentiation performed on the off-resonant image (blue line). (e) Optical microscopy image of the sample composed of micron-sized Cr dots on glass, derived from the "Meisje met de parel" (J. Vermeer, ca. 1665, collection Mauritshuis, The Hague, Netherlands). (f, g) Metasurface output for resonant and off-resonant excitation. The black spot in part g covers an artifact due to spurious reflection at the interface between air and the sapphire substrate. The AMOLF logo and the "Meisje met de parel" painting are reproduced with permission.

(1) Glass slides $\left(24 \times 24 \mathrm{~mm}^{2}\right)$ were cleaned in base piranha.

(2) A bilayer of MMA (MMA(8.5)MAA EL9, $150 \mathrm{~nm}$ ) and PMMA (PMMA 950k A8, $95 \mathrm{~nm}$ ) was spin-coated and baked at 150 and $180{ }^{\circ} \mathrm{C}$ for 2 min, respectively.

(3) The images were fabricated in the resist layer by exposure using a Raith Voyager lithography system (50 $\mathrm{kV}$, dose $550 \mu \mathrm{C} / \mathrm{cm}^{2}$ ) and development in MIBK:IPA (1:3 for $90 \mathrm{~s})$.

(4) A $40 \mathrm{~nm}$ thick Cr layer was evaporated with an in-housebuilt thermal evaporator.

(5) The residual resist was lifted off in anisole at $65{ }^{\circ} \mathrm{C}$.

\section{ASSOCIATED CONTENT}

\section{S Supporting Information}

The Supporting Information is available free of charge on the ACS Publications website at DOI: 10.1021/acs.nanolett.9b02477.
We provide an intuitive explanation of our design strategy supporting it with coupled mode theory and simulations. We explain the asymmetry requirements necessary to design metasurfaces performing oddsymmetry operations, such as first-order spatial differentiation. Next, we give a detailed description of the optical setups used to perform the measurements shown in the main text and an estimate of the metasurface's bandwidth of operation. Finally, we show the complete (real and imaginary part) calculated response of the metasurface compared to its ideal counterpart and an illustration of a possible practical implementation on an imaging system. (PDF)

\section{AUTHOR INFORMATION}

\section{Corresponding Author}

*E-mail: a.polman@amolf.nl.

ORCID ( )

Andrea Cordaro: 0000-0003-3000-7943 
A. Femius Koenderink: 0000-0003-1617-5748

Andrea Alù: 0000-0002-4297-5274

Albert Polman: 0000-0002-0685-3886

\section{Author Contributions}

A.C. designed and fabricated $\mathrm{Si}$ metasurface samples, performed numerical simulations, and performed optical measurements. A.C., H.K., and D.S. performed theoretical analyses. A.F.K., A.A., and A.P. supervised the project. All authors contributed to the analysis and writing of the paper.

\section{Notes}

The authors declare no competing financial interest.

\section{ACKNOWLEDGMENTS}

This work is part of the research program of The Netherlands Organization for Scientific Research (NWO), supported by the European Research Council and by the AFOSR MURI with Grant No. FA9550-17-1-0002.

\section{REFERENCES}

(1) Athale, R.; Psaltis, D. Optical Computing: Past and Future. Opt. Photonics News 2016, 27 (6), 32.

(2) Cutrona, L. J.; Leith, E. N.; Porcello, L. J.; Vivian, W. E. On the Application of Coherent Optical Processing Techniques to SyntheticAperture Radar. Proc. IEEE 1966, 54 (8), 1026-1032.

(3) Abu-Mostafa, Y. S.; Psaltis, D. Optical Neural Computers. Sci. Am. 1987, 256 (3), 88-95.

(4) Silva, A.; Monticone, F.; Castaldi, G.; Galdi, V.; Alu, A.; Engheta, N. Performing Mathematical Operations with Metamaterials. Science 2014, 343 (6167), 160-163.

(5) Kwon, H.; Sounas, D.; Cordaro, A.; Polman, A.; Alù, A. Nonlocal Metasurfaces for Optical Signal Processing. Phys. Rev. Lett. 2018, 121 (17), 173004.

(6) Guo, C.; Xiao, M.; Minkov, M.; Shi, Y.; Fan, S. Photonic Crystal Slab Laplace Operator for Image Differentiation. Optica 2018, 5 (3), 251.

(7) Zhu, T.; Zhou, Y.; Lou, Y.; Ye, H.; Qiu, M.; Ruan, Z.; Fan, S. Plasmonic Computing of Spatial Differentiation. Nat. Commun. 2017, 8 (May), 15391.

(8) Guo, C.; Xiao, M.; Minkov, M.; Shi, Y.; Fan, S. Isotropic Wavevector Domain Image Filters by a Photonic Crystal Slab Device. J. Opt. Soc. Am. A 2018, 35 (10), 1685.

(9) Roberts, A.; Gómez, D. E.; Davis, T. J. Optical Image Processing with Metasurface Dark Modes. J. Opt. Soc. Am. A 2018, 35 (9), 1575.

(10) Fan, S.; Joannopoulos, J. Analysis of Guided Resonances in Photonic Crystal Slabs. Phys. Rev. B: Condens. Matter Mater. Phys. 2002, 65 (23), 1-8.

(11) Wang, S. S.; Moharam, M. G.; Magnusson, R.; Bagby, J. S. Guided-Mode Resonances in Planar Dielectric-Layer Diffraction Gratings. J. Opt. Soc. Am. A 1990, 7 (8), 1470.

(12) Wang, S. S.; Magnusson, R. Theory and Applications of Guided-Mode Resonance Filters. Appl. Opt. 1993, 32 (14), 2606.

(13) Luk'yanchuk, B.; Zheludev, N. I.; Maier, S. A.; Halas, N. J.; Nordlander, P.; Giessen, H.; Chong, C. T. The Fano Resonance in Plasmonic Nanostructures and Metamaterials. Nat. Mater. 2010, 9 (9), 707-715.

(14) Andreani, L. C.; Agio, M. Photonic Bands and Gap Maps in a Photonic Crystal Slab. IEEE J. Quantum Electron. 2002, 38 (7), 891898.

(15) Miroshnichenko, A. E.; Flach, S.; Kivshar, Y. S. Fano Resonances in Nanoscale Structures. Rev. Mod. Phys. 2010, 82 (3), 2257-2298.

(16) Fano, U. Sullo Spettro Di Assorbimento Dei Gas Nobili Presso Il Limite Dello Spettro d'arco. Nuovo Cimento 1935, 12 (3), 154-161.

(17) Fan, S.; Suh, W.; Joannopoulos, J. D. Temporal Coupled-Mode Theory for the Fano Resonance in Optical Resonators. J. Opt. Soc. Am. A 2003, 20 (3), 569-572.
(18) Haus, H. A. Waves and Fields in Optoelectronics; Prentice-Hall: Englewood Cliffs, NJ, 1984.

(19) Sersic, I.; Tuambilangana, C.; Femius Koenderink, A. Fourier Microscopy of Single Plasmonic Scatterers. New J. Phys. 2011, 13 (8), 083019 .

(20) Brundrett, D. L.; Glytsis, E. N.; Gaylord, T. K. Homogeneous Layer Models for High-Spatial-Frequency Dielectric Surface-Relief Gratings: Conical Diffraction and Antireflection Designs. Appl. Opt. 1994, 33 (13), 2695

(21) Kurvits, J. A.; Jiang, M.; Zia, R. Comparative Analysis of Imaging Configurations and Objectives for Fourier Microscopy. J. Opt. Soc. Am. A 2015, 32 (11), 2082.

(22) Saleh, B. E. A.; Teich, M. C. Fundamentals of Photonics; Wiley Series in Pure and Applied Optics; Wiley: 2007.

(23) Goodman, J. W. Introduction to Fourier Optics, 4th ed.; W. H. Freeman: 2017

(24) Green, M. A. Self-Consistent Optical Parameters of Intrinsic Silicon at $300 \mathrm{~K}$ Including Temperature Coefficients. Sol. Energy Mater. Sol. Cells 2008, 92 (11), 1305-1310.

(25) Malitson, I. H.; Dodge, M. J. Refractive Index and Birefringence of Synthetic Sapphire. In Program of the 1972 Annual Meeting of the Optical Society of America; 1972; Vol. 62, p 1336. 